\title{
Plattform Qualitätsmedizin: Bekenntnis zur Transparenz in der Qualität
}

Simon Hölzer

Präsident Schweizerische Gesellschaft für Medizincontrolling SGfM
Korrespondenz: Dr. med. Simon Hölzer Schweizerische Gesellschaft für Medizincontrolling Haslerstrasse 21 CH-3008 Bern Tel. 0313100555 Fax 0313100557

sekretariat[at] medizincontroller.ch
Im Rahmen der Veranstaltungsreihe «Qualitätsmedizin Schweiz» lud am 24. Juni 2014 die Schweizerische Gesellschaft für Medizincontrolling Akteure aus verschiedenen Bereichen des Gesundheitswesens in den Kursaal nach Bern ein. Im Zentrum der sechs Referate und Diskussionsrunden stand das Informationsbedürfnis der Patienten nach verständlichen und verlässlichen Orientierungshilfen. Die hierfür nötige Transparenz der Spitäler wird noch unterschiedlich gelebt, sollte jedoch in jeder Hinsicht positiv, unter anderem auch als Qualitätssicherungs- und Marketinginstrument, verstanden werden. Da der Gesetzgeber den Wettbewerb im Gesundheitswesen stärken möchte, ist es wichtig, nicht nur minimale Anforderungen an die Qualität zu definieren. Erst mit Qualitätsdaten nach einheitlichen Kriterien und mehr Transparenz kann es einen echten Qualitätsvergleich und Qualitätswettbewerb geben.
Besson. Einen genauso grossen Stellenwert räumt Christian Westerhoff, Hirslanden Kliniken, dem Informationsaustausch zu den Qualitätsergebnissen innerhalb der Ärzteschaft ein. Der Qualitätsbericht sollte hier fester Bestandteil einer jeden Klinik sein, genauso wie ein strukturierter Austausch in Netzwerken oder eine regelmässige Erhebung zur Patientenzufriedenheit. «Die Veröffentlichung all dieser Ergebnisse ist ein klares Bekenntnis zur Transparenz in der Qualität und führt nachhaltig zur Qualitätsverbesserung», erklärte Christian Westerhoff die Notwendigkeit eines guten Qualitätsmanagements in den Spitälern.

Bevor man über eventuelle Spitalrankings diskutieren könne, müssten beim Patienten verschiedene Daten zu Behandlungsfehlern, Infektionen oder Wiederaufnahmen abgefragt werden. «Nichts

\section{«Erst mit Qualitätsdaten nach einheitlichen Kriterien und mehr Transparenz kann es einen echten Qualitätsvergleich und Qualitäts- wettbewerb geben.»}

Barbara Miller von der Fachhochschule Nordwestschweiz verdeutlichte den knapp 100 Fachleuten, dass die behandelnden Ärzte die wichtigste Informationsquelle für Patienten sind, noch vor Bekannten und Verwandten, um sich bei einem notwendigen Eingriff für ein bestimmtes Spital zu entscheiden. Aus diesem Grund kommt der Zuweiserpflege in den Spitälern eine ganz besondere Bedeutung zu. «Internationale Studien haben gezeigt, dass die Patientenzufriedenheit direkt mit dem medizinischen Ergebnis korreliert», betonte Barbara Miller. Somit sind Zuweiser die Keyaccounts der Spitäler und die Kommunikationspflege gehört zum Jobprofil von leitenden Klinikärzten. Die Patientenzufriedenheit gilt es in den Vordergrund zu rücken. Sie beginnt schon, wenn potentielle Patienten als Besucher das Spital kennenlernen.

Damit Patienten sich online informieren können, hat der Spitalverband H+ im Spital- und Klinikmonitor verschiedene Informationen zusammengetragen: «Wir möchten möglichst transparent, verlässlich und vollständig Gesundheitsinformationen bereitstellen, damit sich Patienten und Zuweiser vor einem Spitalaufenthalt informieren können», erklärte Pascal ist teurer als schlechte Qualität», warnte dann auch Felix Schneuwly von Comparis.

Ein besonderes Augenmerk legte Thomas Straubhaar vom Nationalen Verein für Qualitätsentwicklung in Spitälern und Kliniken (ANQ) auf die Implementierung eines einheitlichen Messsystems zur Dokumentation der Outcome-Qualität. Hierdurch sollen einheitliche und national vergleichbare Auswertungen erstellt und transparent publiziert werden. Nur so könnten kontinuierliche Verbesserungsprozesse für Spitäler und Kliniken erreicht werden. «Das Thema Qualität ist nie abgeschlossen!», betonte Thomas Straubhaar.

Es ist eine grosse Herausforderung für die nächsten Jahre, die vergleichende Qualitätsmessung und Darstellung noch stärker ins Bewusstsein der Klinikverantwortlichen zu holen. Hier ist Sorge zu tragen, dass sich der Patient gezielt informieren kann bzw. offen informiert wird und sich in «seinem» Spital letztlich aufgehoben fühlt.

Die nächste Tagung «Qualitätsmedizin» findet am Donnerstag, dem 11. Juni 2015, statt und wird eine auf Qualitätsoptimierung ausgerichtete Unternehmenskultur behandeln. 